\title{
The Alperin-McKay Conjecture for metacyclic, minimal non-abelian defect groups
}

\author{
Benjamin Sambale
}

September 13, 2021

\begin{abstract}
We prove the Alperin-McKay Conjecture for all $p$-blocks of finite groups with metacyclic, minimal nonabelian defect groups. These are precisely the metacyclic groups whose derived subgroup have order $p$. In the special case $p=3$, we also verify Alperin's Weight Conjecture for these defect groups. Moreover, in case $p=5$ we do the same for the non-abelian defect groups $C_{25} \rtimes C_{5^{n}}$. The proofs do not rely on the classification of the finite simple groups.
\end{abstract}

Keywords: Alperin-McKay Conjecture, metacyclic defect groups

AMS classification: 20C15, 20C20

\section{Introduction}

Let $B$ be a $p$-block of a finite group $G$ with respect to an algebraically closed field of characteristic $p$. Suppose that $B$ has a metacyclic defect group $D$. We are interested in the number $k(B)$ (respectively $k_{i}(B)$ ) of irreducible characters of $B$ (of height $i \geq 0$ ), and the number $l(B)$ of irreducible Brauer characters of $B$. If $p=2$, these invariants are well understood and the major conjectures are known to be true by work of several authors (see [4, 31, 35, 37, 11, 9]). Thus we will focus on the case $p>2$ in the present work. Here at least Brauer's $k(B)$-Conjecture, Olsson's Conjecture and Brauer's Height Zero Conjecture are satisfied for $B$ (see [14, 43, 38]). By a result of Stancu [40, $B$ is a controlled block. Moreover, if $D$ is a non-split extension of two cyclic groups, it is known that $B$ is nilpotent (see [7]). Then a result by Puig [33 describes the source algebra of $B$ in full detail. Thus we may assume in the following that $D$ is a split extension of two cyclic groups. A famous theorem by Dade [6] handles the case where $D$ itself is cyclic by making use of Brauer trees. The general situation is much harder - even the case $D \cong C_{3} \times C_{3}$ is still open (see [24, 42, 26, 25]). Now consider the subcase where $D$ is non-abelian. Then a work by An [1] shows that $G$ is not a quasisimple group. On the other hand, the algebra structure of $B$ in the $p$-solvable case can be obtained from Külshammer [27]. If $B$ has maximal defect (i. e. $D \in \operatorname{Syl}_{p}(G)$ ), the block invariants of $B$ were determined in [15]. If $B$ is the principal block, Horimoto and Watanabe [20] constructed a perfect isometry between $B$ and its Brauer correspondent in $\mathrm{N}_{G}(D)$.

Let us suppose further that $D$ is a split extension of a cyclic group and a group of order $p$ (i. e. $D$ is the unique non-abelian group with a cyclic subgroup of index $p)$. Here the difference $k(B)-l(B)$ is known from [16. Moreover, under additional assumptions on $G$, Holloway, Koshitani and Kunugi [19] obtained the block invariants precisely. In the special case where $D$ has order $p^{3}$, incomplete information are given by Hendren [17]. Finally, one has full information in case $|D|=27$ by work of the present author [38].

In the present work we consider the following class of non-abelian split metacyclic groups

$$
D=\left\langle x, y \mid x^{p^{m}}=y^{p^{n}}=1, y x y^{-1}=x^{1+p^{m-1}}\right\rangle \cong C_{p^{m}} \rtimes C_{p^{n}}
$$

where $m \geq 2$ and $n \geq 1$. By a result of Rédei (see [21, Aufgabe III.7.22]) these are precisely the metacyclic, minimal non-abelian groups. A result by Knoche (see [21, Aufgabe III.7.24]) implies further that these are exactly the metacyclic groups with derived subgroup of order $p$. In particular the family includes the nonabelian group with a cyclic subgroup of index $p$ mentioned above. The main theorem of the present paper states 
that $k_{0}(B)$ is locally determined. In particular the Alperin-McKay Conjecture holds for $B$. This improves some of the results mentioned above. We also prove that every irreducible character of $B$ has height 0 or 1 . This is in accordance with the situation in $\operatorname{Irr}(D)$. In the second part of the paper we investigate the special case $p=3$. Here we are able to determine $k(B), k_{i}(B)$ and $l(B)$. This gives an example of Alperin's Weight Conjecture and the Ordinary Weight Conjecture. Finally, we determine the block invariants for $p=5$ and $D \cong C_{25} \rtimes C_{5^{n}}$ where $n \geq 1$.

As a new ingredient (compared to [38]) we make use of the focal subgroup of $B$.

\section{The Alperin-McKay Conjecture}

Let $p$ be an odd prime, and let $B$ be a $p$-block with split metacyclic, non-abelian defect group $D$. Then $D$ has a presentation of the form

$$
D=\left\langle x, y \mid x^{p^{m}}=y^{p^{n}}=1, y x y^{-1}=x^{1+p^{l}}\right\rangle
$$

where $0<l<m$ and $m-l \leq n$. Elementary properties of $D$ are stated in the following lemma.

\section{Lemma 2.1.}

(i) $D^{\prime}=\left\langle x^{p^{l}}\right\rangle \cong C_{p^{m-l}}$.

(ii) $\mathrm{Z}(D)=\left\langle x^{p^{m-l}}\right\rangle \times\left\langle y^{p^{m-l}}\right\rangle \cong C_{p^{l}} \times C_{p^{n-m+l}}$.

Proof. Omitted.

We fix a Sylow subpair $\left(D, b_{D}\right)$ of $B$. Then the conjugation of subpairs $\left(Q, b_{Q}\right) \leq\left(D, b_{D}\right)$ forms a saturated fusion system $\mathcal{F}$ on $D$ (see [2]). Here $Q \leq D$ and $b_{Q}$ is a uniquely determined block of $\mathrm{C}_{G}(Q)$. We also have subsections $\left(u, b_{u}\right)$ where $u \in D$ and $b_{u}:=b_{\langle u\rangle}$. By [40], $\mathcal{F}$ is controlled. Moreover by Theorem 2.5 in 14 we may assume that the inertial group of $B$ has the form $\mathrm{N}_{G}\left(D, b_{D}\right) / \mathrm{C}_{G}(D)=\operatorname{Aut}_{\mathcal{F}}(D)=\langle\operatorname{Inn}(D), \alpha\rangle$ where $\alpha \in \operatorname{Aut}(D)$ such that $\alpha(x) \in\langle x\rangle$ and $\alpha(y)=y$. By a slight abuse of notation we often write $\operatorname{Out}_{\mathcal{F}}(D)=\langle\alpha\rangle$. In particular the inertial index $e(B):=\left|\operatorname{Out}_{\mathcal{F}}(D)\right|$ is a divisor of $p-1$. Let

$$
\mathfrak{f o c}(B):=\left\langle f(a) a^{-1}: a \in Q \leq D, f \in \operatorname{Aut}_{\mathcal{F}}(Q)\right\rangle
$$

be the focal subgroup of $B$ (or of $\mathcal{F}$ ). Then it is easy to see that $\mathfrak{f o c}(B) \subseteq\langle x\rangle$. In case $e(B)=1, B$ is nilpotent and $\mathfrak{f o c}(B)=D^{\prime}$. Otherwise $\mathfrak{f o c}(B)=\langle x\rangle$.

For the convenience of the reader we collect some estimates on the block invariants of $B$.

Proposition 2.2. Let $B$ be as above. Then

$$
\begin{aligned}
\left(\frac{p^{l}+p^{l-1}-p^{2 l-m-1}-1}{e(B)+e(B)) p^{n}} \leq k(B) \leq\left(\frac{p^{l}-1}{e(B)}+e(B)\right)\left(p^{n+m-l-2}+p^{n}-p^{n-2}\right),\right. \\
2 p^{n} \leq k_{0}(B) \leq\left(\frac{p^{l}-1}{e(B)}+e(B)\right) p^{n}, \\
\sum_{i=0}^{\infty} p^{2 i} k_{i}(B) \leq\left(\frac{p^{l}-1}{e(B)}+e(B)\right) p^{n+m-l}, \\
l(B) \geq e(B) \mid p-1, \quad \text { for } i \geq 1, \\
p^{n}\left|k_{0}(B), \quad p^{n-m+l}\right| k_{i}(B) \quad \text { for } i>2(m-l) . \\
k_{i}(B)=0 \quad \text { i }
\end{aligned}
$$

Proof. Most of the inequalities are contained in Proposition 2.1 to Corollary 2.5 in 38. By Theorem 1 in 36 we have $p^{n}|| D: \mathfrak{f o c}(B)|| k_{0}(B)$. In particular $p^{n} \leq k_{0}(B)$. In case $k_{0}(B)=p^{n}$ it follows from [23, that $B$ is nilpotent. However then we would have $k_{0}(B)=\left|D: D^{\prime}\right|=p^{n+l}>p^{n}$. Therefore $2 p^{n} \leq k_{0}(B)$. Theorem 2 in [36] implies $p^{n-m+l}|| \mathrm{Z}(D): \mathrm{Z}(D) \cap \mathfrak{f o c}(B)|| k_{i}(B)$ for $i \geq 1$. 
Now we consider the special case where $m=l+1$. As mentioned in the introduction these are precisely the metacyclic, minimal non-abelian groups. We prove the main theorem of this section.

Theorem 2.3. Let $B$ be a p-block of a finite group with metacyclic, minimal non-abelian defect groups for an odd prime $p$. Then

$$
k_{0}(B)=\left(\frac{p^{m-1}-1}{e(B)}+e(B)\right) p^{n}
$$

with the notation from (1.1). In particular the Alperin-McKay Conjecture holds for B.

Proof. By Proposition 2.2 we have

$$
p^{n} \mid k_{0}(B) \leq\left(\frac{p^{m-1}-1}{e(B)}+e(B)\right) p^{n} .
$$

Thus, by way of contradiction we may assume that

$$
k_{0}(B) \leq\left(\frac{p^{m-1}-1}{e(B)}+e(B)-1\right) p^{n} .
$$

We also have

$$
k(B) \geq\left(\frac{p^{m-1}+p^{m-2}-p^{m-3}-1}{e(B)}+e(B)\right) p^{n}
$$

from Proposition 2.2. Hence the sum $\sum_{i=0}^{\infty} p^{2 i} k_{i}(B)$ will be small if $k_{0}(B)$ is large and $k_{1}(B)=k(B)-k_{0}(B)$. This implies the following contradiction

$$
\begin{aligned}
\left(\frac{p^{m}-1}{e(B)}+p^{2}+e(B)-1\right) p^{n} & =\left(\frac{p^{m-1}-1}{e(B)}+e(B)-1\right) p^{n}+\left(\frac{p^{m-2}-p^{m-3}}{e(B)}+1\right) p^{n+2} \\
& \leq \sum_{i=0}^{\infty} p^{2 i} k_{i}(B) \leq\left(\frac{p^{m}-p}{e(B)}+p e(B)\right) p^{n}<\left(\frac{p^{m}-1}{e(B)}+p^{2}\right) p^{n}
\end{aligned}
$$

Since the Brauer correspondent of $B$ in $\mathrm{N}_{G}(D)$ has the same fusion system, the Alperin-McKay Conjecture follows.

Isaacs and Navarro [22, Conjecture D] proposed a refinement of the Alperin-McKay Conjecture by invoking Galois automorphisms. We show (as a improvement of Theorem 4.3 in [38]) that this conjecture holds in the special case $|D|=p^{3}$ of Theorem 2.3. We will denote the subset of $\operatorname{Irr}(B)$ of characters of height 0 by $\operatorname{Irr}_{0}(B)$.

Corollary 2.4. Let $B$ be a p-block of a finite group $G$ with non-abelian, metacyclic defect group of order $p^{3}$. Then Conjecture D in [22] holds for $B$.

Proof. Let $D$ be a defect group of $B$. For $k \in \mathbb{N}$, let $\mathbb{Q}_{k}$ be the cyclotomic field of degree $k$. Let $|G|_{p^{\prime}}$ be the $p^{\prime}$ part of the order of $G$. It is well-known that the Galois group $\mathcal{G}:=\operatorname{Gal}\left(\mathbb{Q}_{|G|} \mid \mathbb{Q}_{|G|_{p^{\prime}}}\right)$ acts canonically on $\operatorname{Irr}(B)$. Let $\gamma \in \mathcal{G}$ be a $p$-element. Then it suffices to show that $\gamma$ acts trivially on $\operatorname{Irr}_{0}(B)$. By Lemma IV.6.10 in [12] it is enough to prove that $\gamma$ acts trivially on the $\mathcal{F}$-conjugacy classes of subsections of $B$ via ${ }^{\gamma}\left(u, b_{u}\right):=\left(u^{\bar{\gamma}}, b_{u}\right)$ where $u \in D$ and $\bar{\gamma} \in \mathbb{Z}$. Since $\gamma$ is a $p$-element, this action is certainly trivial unless $|\langle u\rangle|=p^{2}$. Here however, the action of $\gamma$ on $\langle u\rangle$ is just the $D$-conjugation. The result follows.

In the situation of Corollary 2.4 one can say a bit more: By Proposition 3.3 in [38, $\operatorname{Irr}(B)$ splits into the following families of $p$-conjugate characters:

- $(p-1) / e(B)+e(B)$ orbits of length $p-1$,

- two orbits of length $(p-1) / e(B)$,

- at least $e(B) p$-rational characters. 
Without loss of generality, let $e(B)>1$. By Theorem 4.1 in 38 we have $k_{1}(B) \leq(p-1) / e(B)+e(B)-1$. Moreover, Proposition 4.1 of the same paper implies $k_{1}(B)<p-1$. In particular, all orbits of length $p-1$ of $p$-conjugate characters must lie in $\operatorname{Irr}_{0}(B)$. In case $e(B)=p-1$ the remaining $(p-1) / e(B)+e(B)$ characters in $\operatorname{Irr}_{0}(B)$ must be $p$-rational. Now let $e(B)<\sqrt{p-1}$. Then it is easy to see that $\operatorname{Irr}_{0}(B)$ contains just one orbit of length $(p-1) / e(B)$ of $p$-conjugate characters. Unfortunately, it is not clear if this also holds for $e(B) \geq \sqrt{p-1}$.

Next we improve the bound coming from Proposition 2.2 on the heights of characters.

Proposition 2.5. Let $B$ be a p-block of a finite group with metacyclic, minimal non-abelian defect groups. Then $k_{1}(B)=k(B)-k_{0}(B)$. In particular, $B$ satisfies the following conjectures:

- Eaton's Conjecture [8]

- Eaton-Moretó Conjecture [10]

- Robinson's Conjecture [28, Conjecture 4.14.7]

- Malle-Navarro Conjecture [29]

Proof. By [37] we may assume $p>2$ as before. By way of contradiction suppose that $k_{i}(B)>0$ for some $i \geq 2$. Since

$$
k(B) \geq\left(\frac{p^{m-1}+p^{m-2}-p^{m-3}-1}{e(B)}+e(B)\right) p^{n},
$$

we have $k(B)-k_{0}(B) \geq\left(p^{m-1}-p^{m-2}\right) p^{n-1} / e(B)$ by Theorem 2.3. By Proposition 2.2. $k_{1}(B)$ and $k_{i}(B)$ are divisible by $p^{n-1}$. This shows

$$
\left(\frac{p^{m-1}-1}{e(B)}+e(B)\right) p^{n}+\left(\frac{p^{m-1}-p^{m-2}}{e(B)}-1\right) p^{n+1}+p^{n+3} \leq \sum_{i=0}^{\infty} p^{2 i} k_{i}(B) \leq\left(\frac{p^{m-1}-1}{e(B)}+e(B)\right) p^{n+1} .
$$

Hence, we derive the following contradiction

$$
p^{n+3}-p^{n+1} \leq\left(\frac{1-p}{e(B)}+e(B)(p-1)\right) p^{n} \leq p^{n+2}
$$

This shows $k_{1}(B)=k(B)-k_{0}(B)$. Now Eaton's Conjecture is equivalent to Brauer's $k(B)$-Conjecture and Olsson's Conjecture. Both are known to hold for all metacyclic defect groups. Also the Eaton-Moretó Conjecture and Robinson's Conjecture is trivially satisfied for $B$. The Malle-Navarro Conjecture asserts that $k(B) / k_{0}(B) \leq$ $k\left(D^{\prime}\right)=p$ and $k(B) / l(B) \leq k(D)$. By Theorem 2.3 and Proposition 2.2, the first inequality reduces to $p^{n-1}+$ $p^{n}-p^{n-2} \leq p^{n+1}$ which is true. For the second inequality we observe that every conjugacy class of $D$ has at most $p$ elements, since $|D: \mathrm{Z}(D)|=p^{2}$. Hence, $k(D)=|\mathrm{Z}(D)|+\frac{|D|-|\mathrm{Z}(D)|}{p}=p^{n+m-1}+p^{n+m-2}-p^{n+m-3}$. Now Proposition 2.2 gives

$$
\frac{k(B)}{l(B)} \leq k(B) \leq\left(\frac{p^{m-1}-1}{e(B)}+e(B)\right)\left(p^{n-1}+p^{n}-p^{n-2}\right) \leq p^{n+m-1}+p^{n+m-2}-p^{n+m-3}=k(D) .
$$

We use the opportunity to present a result for $p=3$ and a different class of metacyclic defect groups (where $l=1$ with the notation above).

Theorem 2.6. Let $B$ be a 3-block of a finite group $G$ with defect group

$$
D=\left\langle x, y \mid x^{3^{m}}=y^{3^{n}}=1, y x y^{-1}=x^{4}\right\rangle
$$

where $2 \leq m \leq n+1$. Then $k_{0}(B)=3^{n+1}$. In particular, the Alperin-McKay Conjecture holds for B.

Proof. We may assume that $B$ is non-nilpotent. By Proposition 2.2 we have $k_{0}(B) \in\left\{2 \cdot 3^{n}, 3^{n+1}\right\}$. By way of contradiction, suppose that $k_{0}(B)=2 \cdot 3^{n}$. Let $P \in \operatorname{Syl}_{p}(G)$. Since $D / \mathfrak{f o c}(B)$ acts freely on $\operatorname{Irr}_{0}(B)$, there are $3^{n}$ characters of degree $a|P: D|$, and $3^{n}$ characters of degree $b|P: D|$ in $B$ for some $a, b \geq 1$ such that $3 \nmid a, b$. Hence,

$$
\left|\sum_{\chi \in \operatorname{Irr}_{0}(B)} \chi(1)^{2}\right|_{3}=3^{n}|P: D|^{2}\left(a^{2}+b^{2}\right)_{3}=|P: D|^{2}|D: \mathfrak{f o c}(B)| .
$$

Now Theorem 1.1 in 23] gives a contradiction. 
A generalization of the argument in the proof shows that in the situation of Proposition $2.2, k_{0}(B)=2 p^{n}$ can only occur if $p \equiv 1(\bmod 4)$.

\section{Lower defect groups}

In the following we use the theory of lower defect groups in order to estimate $l(B)$. We cite a few results from the literature. Let $B$ be a $p$-block of a finite group $G$ with defect group $D$ and Cartan matrix $C$. We denote the multiplicity of an integer $a$ as elementary divisor of $C$ by $m(a)$. Then $m(a)=0$ unless $a$ is a $p$-power. It is well-known that $m(|D|)=1$. Brauer [3] expressed $m\left(p^{n}\right)(n \geq 0)$ in terms of 1-multiplicities of lower defect groups (see also Corollary V.10.12 in [12]):

$$
m\left(p^{n}\right)=\sum_{R \in \mathcal{R}} m_{B}^{(1)}(R)
$$

where $\mathcal{R}$ is a set of representatives for the $G$-conjugacy classes of subgroups $R \leq D$ of order $p^{n}$. Later (3.1) was refined by Broué and Olsson by invoking the fusion system $\mathcal{F}$ of $B$.

Proposition 3.1 (Broué-Olsson [5]). For $n \geq 0$ we have

$$
m\left(p^{n}\right)=\sum_{R \in \mathcal{R}} m_{B}^{(1)}\left(R, b_{R}\right)
$$

where $\mathcal{R}$ is a set of representatives for the $\mathcal{F}$-conjugacy classes of subgroups $R \leq D$ of order $p^{n}$.

Proof. This is $(2 \mathrm{~S})$ of $[5]$.

In the present paper we do not need the precise (and complicated) definition of the non-negative numbers $m_{B}^{(1)}(R)$ and $m_{B}^{(1)}\left(R, b_{R}\right)$. We say that $R$ is a lower defect group for $B$ if $m_{B}^{(1)}\left(R, b_{R}\right)>0$. In particular, $m_{B}^{(1)}\left(D, b_{D}\right)=m_{B}^{(1)}(D)=m(|D|)=1$. A crucial property of lower defect groups is that their multiplicities can usually be determined locally. In the next lemma, $b_{R}^{\mathrm{N}_{G}\left(R, b_{R}\right)}$ denotes the (unique) Brauer correspondent of $b_{R}$ in $\mathrm{N}_{G}\left(R, b_{R}\right)$.

Lemma 3.2. For $R \leq D$ and $B_{R}:=b_{R}^{\mathrm{N}_{G}\left(R, b_{R}\right)}$ we have $m_{B}^{(1)}\left(R, b_{R}\right)=m_{B_{R}}^{(1)}(R)$. If $R$ is fully $\mathcal{F}$-normalized, then $B_{R}$ has defect group $\mathrm{N}_{D}(R)$ and fusion system $\mathrm{N}_{\mathcal{F}}(R)$.

Proof. The first claim follows from (2Q) in [5]. For the second claim we refer to Theorem IV.3.19 in [2].

Another important reduction is given by the following lemma.

Lemma 3.3. For $R \leq D$ we have $\sum_{Q \in \mathcal{R}} m_{B_{R}}^{(1)}(Q) \leq l\left(b_{R}\right)$ where $\mathcal{R}$ is a set of representatives for the $\mathrm{N}_{G}\left(R, b_{R}\right)$ conjugacy classes of subgroups $Q$ such that $R \leq Q \leq \mathrm{N}_{D}(R)$.

Proof. This is implied by Theorem 5.11 in 32 and the remark following it. Notice that in Theorem 5.11 it should read $B \in \mathrm{Bl}(G)$ instead of $B \in \mathrm{Bl}(Q)$.

In the local situation for $B_{R}$ also the next lemma is useful.

Lemma 3.4. If $\mathrm{O}_{p}(\mathrm{Z}(G)) \nsubseteq R$, then $m_{B}^{(1)}(R)=0$.

Proof. See Corollary 3.7 in 32 .

Now we apply these results.

Lemma 3.5. Let $B$ be a p-block of a finite group with metacyclic, minimal non-abelian defect group $D$ for an odd prime $p$. Then every lower defect group of $B$ is D-conjugate either to $\langle y\rangle,\left\langle y^{p}\right\rangle$, or to $D$. 
Proof. Let $R<D$ be a lower defect group of $B$. Then $m(|R|)>0$ by Proposition 3.1. Corollary 5 in 36 shows that $p^{n-1}|| R \mid$. Since $\mathcal{F}$ is controlled, the subgroup $R$ is fully $\mathcal{F}$-centralized and fully $\mathcal{F}$-normalized. The fusion system of $b_{R}\left(\right.$ on $\left.\mathrm{C}_{D}(R)\right)$ is given by $\mathrm{C}_{\mathcal{F}}(R)$ (see Theorem IV.3.19 in [2]). Suppose for the moment that $\mathrm{C}_{\mathcal{F}}(R)$ is trivial. Then $b_{R}$ is nilpotent and $l\left(b_{R}\right)=1$. Let $B_{R}:=b_{R}^{\mathrm{N}_{G}\left(R, b_{R}\right)}$. Then $B_{R}$ has defect group $\mathrm{N}_{D}(R)$ and $m_{B_{R}}^{(1)}\left(\mathrm{N}_{D}(R)\right)=1$. Hence, Lemmas 3.2 and 3.3 imply $m_{B}^{(1)}\left(R, b_{R}\right)=m_{B_{R}}^{(1)}(R)=0$. This contradiction shows that $\mathrm{C}_{\mathcal{F}}(R)$ is non-trivial. In particular $R$ is centralized by a non-trivial $p^{\prime}$-automorphism $\beta \in \operatorname{Aut} \mathcal{F}(D)$. By the Schur-Zassenhaus Theorem, $\beta$ is $\operatorname{Inn}(D)$-conjugate to a power of $\alpha$. Thus, $R$ is $D$-conjugate to a subgroup of $\langle y\rangle$. The result follows.

Proposition 3.6. Let $B$ be a p-block of a finite group with metacyclic, minimal non-abelian defect groups for an odd prime $p$. Then $e(B) \leq l(B) \leq 2 e(B)-1$.

Proof. Let

$$
D=\left\langle x, y \mid x^{p^{m}}=y^{p^{n}}=1, y x y^{-1}=x^{1+p^{m-1}}\right\rangle
$$

be a defect group of $B$. We argue by induction on $n$. Let $n=1$. By Proposition 2.2 we have $e(B) \leq l(B)$ and

$$
k(B) \leq\left(\frac{p^{m-1}-1}{e(B)}+e(B)\right)\left(1+p-p^{-1}\right) .
$$

Moreover, Theorem 3.2 in [38] gives

$$
k(B)-l(B)=\frac{p^{m}+p^{m-1}-p^{m-2}-p}{e(B)}+e(B)(p-1)
$$

Hence,

$$
\begin{aligned}
l(B) & =k(B)-(k(B)-l(B)) \leq\left(\frac{p^{m-1}-1}{e(B)}+e(B)\right)\left(1+p-p^{-1}\right)-\frac{p^{m}+p^{m-1}-p^{m-2}-p}{e(B)}-e(B)(p-1) \\
& =2 e(B)-\frac{1}{p}\left(e(B)-\frac{1}{e(B)}\right)-\frac{1}{e(B)}
\end{aligned}
$$

and the claim follows in this case.

Now suppose $n \geq 2$. We determine the multiplicities of the lower defect groups by using Lemma 3.5 As usual $m(|D|)=1$. Consider the subpair $\left(\langle y\rangle, b_{y}\right)$. By Lemmas 3.1 and 3.2 we have $m\left(p^{n}\right)=m_{B}^{(1)}\left(\langle y\rangle, b_{y}\right)=m_{B_{y}}^{(1)}(\langle y\rangle)$ where $B_{y}:=b_{y}^{\mathrm{N}_{G}\left(\langle y\rangle, b_{y}\right)}$. Since $\mathrm{N}_{D}(\langle y\rangle)=\mathrm{C}_{D}(y)$, it follows easily that $\mathrm{N}_{G}\left(\langle y\rangle, b_{y}\right)=\mathrm{C}_{G}(y)$ and $B_{y}=b_{y}$. By Theorem IV.3.19 in [2] the block $b_{y}$ has defect group $\mathrm{C}_{D}(y)$ and fusion system $\mathrm{C}_{\mathcal{F}}(\langle y\rangle)$. In particular $e\left(b_{y}\right)=e(B)$. It is well-known that $b_{y}$ dominates a block $\overline{b_{y}}$ of $\mathrm{C}_{G}(y) /\langle y\rangle$ with cyclic defect group $\mathrm{C}_{D}(y) /\langle y\rangle$ and $e\left(\overline{b_{y}}\right)=e\left(b_{y}\right)=e(B)$ (see [30, Theorem 5.8.11]). By Dade's Theorem on blocks with cyclic defect groups we obtain $l\left(b_{y}\right)=e(B)$. Moreover, the Cartan matrix of $\overline{b_{y}}$ has elementary divisors 1 and $\left|\mathrm{C}_{D}(y) /\langle y\rangle\right|$ where 1 occurs with multiplicity $e(B)-1$ (this follows for example from [13]). Therefore, the Cartan matrix of $b_{y}$ has elementary divisors $p^{n}$ and $\left|\mathrm{C}_{D}(y)\right|$ where $p^{n}$ occurs with multiplicity $e(B)-1$. Since $\langle y\rangle \subseteq \mathrm{Z}\left(\mathrm{C}_{G}(y)\right)$, Lemma 3.4 implies $m\left(p^{n}\right)=m_{b_{y}}^{(1)}(\langle y\rangle)=e(B)-1$.

Now consider $\left(\langle u\rangle, b_{u}\right)$ where $u:=y^{p} \in \mathrm{Z}(D)$. Here $b_{u}$ has defect group $D$. By the first part of the proof (the case $n=1)$ we obtain $l\left(b_{u}\right)=l\left(\overline{b_{u}}\right) \leq 2 e(B)-1$. As above we have $m\left(p^{n-1}\right)=m_{B}^{(1)}\left(\langle u\rangle, b_{u}\right)=m_{b_{u}}^{(1)}(\langle u\rangle)$. Since $p^{n}$ occurs as elementary divisor of the Cartan matrix of $b_{u}$ with multiplicity $e(B)-1$ (see above), it follows that $m\left(p^{n-1}\right)=m_{b_{u}}^{(1)}(\langle u\rangle) \leq e(B)-1$. Now $l(B)$ is the sum over the multiplicities of elementary divisors of the Cartan matrix of $B$ which is at most $m(|D|)+m(\langle y\rangle)+m(\langle u\rangle) \leq 1+e(B)-1+e(B)-1=2 e(B)-1$.

The next proposition gives a reduction method.

Proposition 3.7. Let $p>2, m \geq 2$ and $e \mid p-1$ be fixed. Suppose that $l(B)=e$ holds for every block $B$ with defect group

$$
D=\left\langle x, y \mid x^{p^{m}}=y^{p}=1, y x y^{-1}=x^{1+p^{m-1}}\right\rangle
$$


and $e(B)=e$. Then every block $B$ with $e(B)=e$ and defect group

$$
D=\left\langle x, y \mid x^{p^{m}}=y^{p^{n}}=1, y x y^{-1}=x^{1+p^{m-1}}\right\rangle
$$

where $n \geq 1$ satisfies the following:

$$
\begin{aligned}
k_{0}(B) & =\left(\frac{p^{m-1}-1}{e(B)}+e(B)\right) p^{n}, & k_{1}(B) & =\frac{p^{m-1}-p^{m-2}}{e(B)} p^{n-1}, \\
k(B) & =\left(\frac{p^{m}+p^{m-1}-p^{m-2}-p}{e(B)}+e(B) p\right) p^{n-1}, & l(B) & =e(B) .
\end{aligned}
$$

Proof. We use induction on $n$. In case $n=1$ the result follows from Theorem 3.2 in [38], Theorem 2.3] and Proposition 2.5.

Now let $n \geq 2$. Let $\mathcal{R}$ be a set of representatives for the $\mathcal{F}$-conjugacy classes of elements of $D$. We are going to use Theorem 5.9.4 in [30]. For $1 \neq u \in \mathcal{R}, b_{u}$ has metacyclic defect group $\mathrm{C}_{D}(u)$ and fusion $\operatorname{system~} \mathrm{C}_{\mathcal{F}}(\langle u\rangle)$. If $\mathrm{C}_{\mathcal{F}}(\langle u\rangle)$ is non-trivial, $\alpha \in \operatorname{Aut}_{\mathcal{F}}(D)$ centralizes a $D$-conjugate of $u$. Hence, we may assume that $u \in\langle y\rangle$ in this case. If $\langle u\rangle=\langle y\rangle$, then $b_{u}$ dominates a block $\overline{b_{u}}$ of $\mathrm{C}_{G}(u) /\langle u\rangle$ with cyclic defect group $\mathrm{C}_{D}(u) /\langle u\rangle$. Hence, $l\left(b_{u}\right)=l\left(\overline{b_{u}}\right)=e(B)$. Now suppose that $\langle u\rangle\left\langle\langle y\rangle\right.$. Then by induction we obtain $l\left(b_{u}\right)=l\left(\overline{b_{u}}\right)=e(B)$. Finally assume that $\mathrm{C}_{\mathcal{F}}(\langle u\rangle)$ is trivial. Then $b_{u}$ is nilpotent and $l\left(b_{u}\right)=1$. It remains to determine $\mathcal{R}$. The powers of $y$ are pairwise non-conjugate in $\mathcal{F}$. As in the proof of Proposition 2.5. $D$ has precisely $p^{n+m-3}\left(p^{2}+p-1\right)$ conjugacy classes. Let $C$ be one of these classes which do not intersect $\langle y\rangle$. Assume $\alpha^{i}(C)=C$ for some $i \in \mathbb{Z}$ such that $\alpha^{i} \neq 1$. Then there are elements $u \in C$ and $w \in D$ such that $\alpha^{i}(u)=w u w^{-1}$. Hence $\gamma:=w^{-1} \alpha^{i} \in \mathrm{N}_{G}\left(D, b_{D}\right) \cap \mathrm{C}_{G}(u)$. Since $\gamma$ is not a $p$-element, we conclude that $u$ is conjugate to a power of $y$ which was excluded. This shows that no nontrivial power of $\alpha$ can fix $C$ as a set. Thus, all these conjugacy classes split in

$$
\frac{p^{2}+p-p^{3-m}-1}{e(B)} p^{n+m-3}
$$

orbits of length $e(B)$ under the action of $\operatorname{Out}_{\mathcal{F}}(D)$. Now Theorem 5.9.4 in 30] implies

$$
k(B)-l(B)=\left(\frac{p^{m-1}+p^{m-2}-p^{m-3}-1}{e(B)}+e(B)\right) p^{n}-e(B) .
$$

By Proposition 3.6 it follows that

$$
k(B) \leq\left(\frac{p^{m}+p^{m-1}-p^{m-2}-p}{e(B)}+e(B) p\right) p^{n-1}+e(B)-1 .
$$

By Proposition 2.2 the left hand side of (3.2) is divisible by $p^{n-1}$. Since $e(B)-1<p^{n-1}$, we obtain the exact value of $k(B)$. It follows that $l(B)=e(B)$. Finally, Theorem 2.3 and Proposition 2.5 give $k_{i}(B)$.

For $p=3$, Proposition 3.6 implies $l(B) \leq 3$. Here we are able to determine all block invariants.

Theorem 3.8. Let $B$ be a non-nilpotent 3-block of a finite group with metacyclic, minimal non-abelian defect groups. Then

$$
\begin{aligned}
& k_{0}(B)=\frac{3^{m-2}+1}{2} 3^{n+1}, \\
& k_{1}(B)=3^{m+n-3}, \\
& k(B)=\frac{11 \cdot 3^{m-2}+9}{2} 3^{n-1}, \\
& l(B)=e(B)=2
\end{aligned}
$$

with the notation from (1.1).

Proof. By Proposition 3.7 it suffices to settle the case $n=1$. Here the claim holds for $m \leq 3$ by Theorem 3.7 in [38. We will extend the proof of this result in order to handle the remaining $m \geq 4$. Since $B$ is non-nilpotent, we have $e(B)=2$. By Theorem 2.3 we know $k_{0}(B)=\left(3^{m}+9\right) / 2$. By way of contradiction we may assume that $l(B)=3$ and $k_{1}(B)=3^{m-2}+1$ (see Theorem 3.4 in 38 ]). 
We consider the generalized decomposition numbers $d_{\chi \varphi_{z}}^{z}$ where $z:=x^{3} \in \mathrm{Z}(D)$ and $\varphi_{z}$ is the unique irreducible Brauer character of $b_{z}$. Let $d^{z}:=\left(d_{\chi \varphi_{z}}^{z}: \chi \in \operatorname{Irr}(B)\right)$. By the orthogonality relations we have $\left(d^{z}, d^{z}\right)=3^{m+1}$. As in [18, Section 4] we can write

$$
d^{z}=\sum_{i=0}^{2 \cdot 3^{m-2}-1} a_{i} \zeta_{3^{m-1}}^{i}
$$

for integral vectors $a_{i}$ and a primitive $3^{m-1}$-th root of unity $\zeta_{3^{m-1}} \in \mathbb{C}$. Since $z$ is $\mathcal{F}$-conjugate to $z^{-1}$, the vector $d^{z}$ is real. Hence, the vectors $a_{i}$ are linearly dependent. More precisely, it turns out that the vectors $a_{i}$ are spanned by $\left\{a_{j}: j \in J\right\}$ for a subset $J \subseteq\left\{0, \ldots, 2 \cdot 3^{m-2}-1\right\}$ such that $0 \in J$ and $|J|=3^{m-2}$.

Let $q$ be the quadratic form corresponding to the Dynkin diagram of type $A_{3^{m-2}}$. We set $a(\chi):=\left(a_{j}(\chi): j \in J\right)$ for $\chi \in \operatorname{Irr}(B)$. Since the subsection $\left(z, b_{z}\right)$ gives equality in Theorem 4.10 in [18], we have

$$
k_{0}(B)+9 k_{1}(B)=\sum_{\chi \in \operatorname{Irr}(B)} q(a(\chi))
$$

for a suitable ordering of $J$. This implies $q(a(\chi))=3^{2 h(\chi)}$ for $\chi \in \operatorname{Irr}(B)$ where $h(\chi)$ is the height of $\chi$. Moreover, if $a_{0}(\chi) \neq 0$, then $a_{0}(\chi)= \pm 3^{h(\chi)}$ by Lemma 3.6 in [38]. By Lemma 4.7 in [18] we have $\left(a_{0}, a_{0}\right)=27$.

In the next step we determine the number $\beta$ of 3 -rational characters of of height 1 . Since $\left(a_{0}, a_{0}\right)=27$, we have $\beta<4$. On the other hand, the Galois group $\mathcal{G}$ of $\mathbb{Q}\left(\zeta_{\zeta_{3}-1}\right) \cap \mathbb{R}$ over $\mathbb{Q}$ acts on $d^{z}$ and the length of every non-trivial orbit is divisible by 3 (because $\mathcal{G}$ is a 3 -group). This implies $\beta=1$, since $k_{1}(B)=3^{m-2}+1$.

In order to derive a contradiction, we repeat the argument with the subsection $\left(x, b_{x}\right)$. Again we get equality in Theorem 4.10 , but this time for $k_{0}(B)$ instead of $k_{0}(B)+9 k_{1}(B)$. Hence, $d^{x}(\chi)=0$ for characters $\chi \in \operatorname{Irr}(B)$ of height 1. Again we can write $d^{x}=\sum_{i=0}^{2 \cdot 3^{m-1}-1} \overline{a_{i}} \zeta_{3^{m}}^{i}$ where $\overline{a_{i}}$ are integral vectors. Lemma 4.7 in [18] implies $\left(\bar{a}_{0}, \bar{a}_{0}\right)=9$. Using Lemma 3.6 in [38] we also have $\bar{a}_{0}(\chi) \in\{0, \pm 1\}$. By Proposition 3.3 in [38] we have precisely three 3-rational characters $\chi_{1}, \chi_{2}, \chi_{3} \in \operatorname{Irr}(B)$ of height 0 (note that altogether we have four 3-rational characters). Then $a_{0}\left(\chi_{i}\right)= \pm \bar{a}_{0}\left(\chi_{i}\right)= \pm 1$ for $i=1,2,3$. By [36. Section 1] we have $\lambda * \chi_{i} \in \operatorname{Irr}_{0}(B)$ and $\left(\lambda * \chi_{i}\right)(u)=\chi_{i}(u)=d_{\chi_{i} \varphi_{u}}^{u} \varphi_{u}(1)$ for $\lambda \in \operatorname{Irr}(D / \mathfrak{f o c}(B)) \cong C_{3}$ and $u \in\{x, z\}$. Since this action on $\operatorname{Irr}_{0}(B)$ is free, we have nine characters $\psi \in \operatorname{Irr}(B)$ such that $a_{0}(\psi)= \pm \bar{a}_{0}(\psi)= \pm 1$. In particular $\left(a_{0}, \overline{a_{0}}\right) \equiv 1(\bmod 2)$. By the orthogonality relations we have $\left(d^{z}, d^{x^{j}}\right)=0$ for all $j \in \mathbb{Z}$ such that $3 \nmid j$. Using Galois theory we get the final contradiction $0=\left(d^{z}, \bar{a}_{0}\right)=\left(a_{0}, \bar{a}_{0}\right) \equiv 1(\bmod 2)$.

In the smallest case $D \cong C_{9} \rtimes C_{3}$ of Theorem 3.8 even more information on $B$ were given in Theorem 4.5 in [38].

Corollary 3.9. Alperin's Weight Conjecture and the Ordinary Weight Conjecture are satisfied for every 3-block with metacyclic, minimal non-abelian defect groups.

Proof. Let $D$ be a defect group of $B$. Since $B$ is controlled, Alperin's Weight Conjecture asserts that $l(B)=$ $l\left(B_{D}\right)$ where $B_{D}$ is a Brauer correspondent of $B$ in $\mathrm{N}_{G}(D)$. Since both numbers equal $e(B)$, the conjecture holds.

Now we prove the Ordinary Weight Conjecture in the form of [2, Conjecture IV.5.49]. Since Out $\mathcal{F}(D)$ is cyclic, all 2-cocycles appearing in this version are trivial. Therefore the conjecture asserts that $k_{i}(B)$ only depends on $\mathcal{F}$ and thus on $e(B)$. Since the conjecture is known to hold for the principal block of the solvable group $G=D \rtimes C_{e(B)}$, the claim follows.

We remark that Alperin's Weight Conjecture is also true for the abelian defect groups $D \cong C_{3^{n}} \times C_{3^{m}}$ where $n \neq m$ (see [41, 34]).

We observe another consequence for arbitrary defect groups.

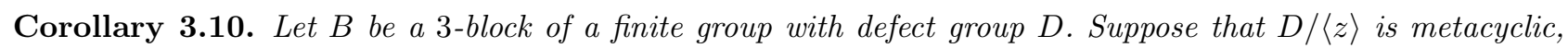
minimal non-abelian for some $z \in \mathrm{Z}(D)$. Then Brauer's $k(B)$-Conjecture holds for $B$, i. e. $k(B) \leq|D|$. 
Proof. Let $\left(z, b_{z}\right)$ be a major subsection of $B$. Then $b_{z}$ dominates a block $\overline{b_{z}}$ of $\mathrm{C}_{G}(z) /\langle z\rangle$ with metacyclic, minimal non-abelian defect group $D /\langle z\rangle$. Hence, Theorem 3.8 implies $l\left(b_{z}\right)=l\left(\overline{b_{z}}\right) \leq 2$. Now the claim follows from Theorem 2.1 in 39 ].

In the situation of Theorem 3.8 it is straight-forward to distribute $\operatorname{Irr}(B)$ into families of 3-conjugate and 3rational characters (cf. Proposition 3.3 in [38]). However, it is not so easy to see which of these families lie in $\operatorname{Irr}_{0}(B)$.

Now we turn to $p=5$.

Theorem 3.11. Let $B$ be a 5-block of a finite group with non-abelian defect group $C_{25} \rtimes C_{5^{n}}$ where $n \geq 1$. Then

$$
\begin{aligned}
k_{0}(B) & =\left(\frac{4}{e(B)}+e(B)\right) 5^{n}, & k_{1}(B) & =\frac{4}{e(B)} 5^{n-1}, \\
k(B) & =\left(\frac{24}{e(B)}+5 e(B)\right) 5^{n-1}, & l(B) & =e(B) .
\end{aligned}
$$

Proof. By Proposition 3.7 it suffices to settle the case $n=1$. Moreover by Theorem 4.4 in [38] we may assume that $e(B)=4$. Then by Theorem 2.3 above and Proposition 4.2 in $\left[38\right.$, we have $k_{0}(B)=25,1 \leq k_{1}(B) \leq 3,26 \leq$ $k(B) \leq 28$ and $4 \leq l(B) \leq 6$. We consider the generalized decomposition numbers $d_{\chi \varphi_{z}}^{z}$ where $z:=x^{5} \in \mathrm{Z}(D)$ and $\varphi_{z}$ is the unique irreducible Brauer character of $b_{z}$. Since all non-trivial powers of $z$ are $\mathcal{F}$-conjugate, the numbers $d_{\chi \varphi_{z}}^{z}$ are integral. Also, these numbers are non-zero, because $\left(z, b_{z}\right)$ is a major subsection. Moreover, $d_{\chi \varphi_{z}}^{z} \equiv 0(\bmod p)$ for characters $\chi \in \operatorname{Irr}(B)$ of height 1 (see Theorem V.9.4 in [12]). Let $d^{z}:=\left(d_{\chi \varphi_{z}}^{z}: \chi \in \operatorname{Irr}(B)\right)$. By the orthogonality relations we have $\left(d^{z}, d^{z}\right)=125$. Suppose by way of contradiction that $k_{1}(B)>1$. Then it is easy to see that $d_{\chi \varphi_{z}}^{z}= \pm 5$ for characters $\chi \in \operatorname{Irr}(B)$ of height 1. By [36, Section 1], the numbers $d_{\chi \varphi_{z}}^{z}$ $\left(\chi \in \operatorname{Irr}_{0}(B)\right)$ split in five orbits of length 5 each. Let $\alpha$ (respectively $\left.\beta, \gamma\right)$ be the number of orbits of entries \pm 1 (respectively $\pm 2, \pm 3$ ) in $d^{z}$. Then the orthogonality relations reads

$$
\alpha+4 \beta+9 \gamma+5 k_{1}(B)=25
$$

Since $\alpha+\beta+\gamma=5$, we obtain

$$
3 \beta+8 \gamma=20-5 k_{1}(B) \in\{5,10\} .
$$

However, this equation cannot hold for any choice of $\alpha, \beta, \gamma$. Therefore we have proved that $k_{1}(B)=1$. Now Theorem 4.1 in 38 implies $l(B)=4$.

Corollary 3.12. Alperin's Weight Conjecture and the Ordinary Weight Conjecture are satisfied for every 5-block with non-abelian defect group $C_{25} \rtimes C_{5^{n}}$.

\section{Proof. See Corollary 3.9}

Unfortunately, the proof of Theorem 3.11 does not work for $p=7$ and $e(B)=6$ (even by invoking the other generalized decomposition numbers). However, we have the following partial result.

Proposition 3.13. Let $p \in\{7,11,13,17,23,29\}$ and let $B$ be a p-block of a finite group with defect group $C_{p^{2}} \rtimes C_{p^{n}}$ where $n \geq 1$. If $e(B)=2$, then

$$
\begin{aligned}
k_{0}(B) & =\frac{p+3}{2} p^{n}, & k_{1}(B) & =\frac{p-1}{2} p^{n-1}, \\
k(B) & =\frac{p^{2}+4 p-1}{2} p^{n-1}, & l(B) & =2 .
\end{aligned}
$$

Proof. We follow the proof of Theorem 4.4 in [38] in order to handle the case $n=1$. After that the result follows from Proposition 3.7. 
In fact the first part of the proof of Theorem 4.4 in [38] applies to any prime $p \geq 7$. Hence, we know that the generalized decomposition numbers $d_{\chi \varphi_{z}}^{z}=a_{0}(\chi)$ for $z:=x^{p}$ and $\chi \in \operatorname{Irr}_{0}(B)$ are integral. Moreover,

$$
\sum_{\chi \in \operatorname{Irr}_{0}(B)} a_{0}(\chi)^{2}=p^{2} .
$$

The action of $D / \mathfrak{f o c}(B)$ on $\operatorname{Irr}_{0}(B)$ shows that the values $a(\chi)$ distribute in $(p+3) / 2$ parts of $p$ equal numbers each. Therefore, Eq. (4.1) in [38] becomes

$$
\sum_{i=2}^{\infty} r_{i}\left(i^{2}-1\right)=\frac{p-3}{2}
$$

for some $r_{i} \geq 0$. This gives a contradiction.

\section{Acknowledgment}

This work is supported by the Carl Zeiss Foundation and the Daimler and Benz Foundation.

\section{References}

[1] J. An, Controlled blocks of the finite quasisimple groups for odd primes, Adv. Math. 227 (2011), 1165-1194.

[2] M. Aschbacher, R. Kessar and B. Oliver, Fusion systems in algebra and topology, London Mathematical Society Lecture Note Series, Vol. 391, Cambridge University Press, Cambridge, 2011.

[3] R. Brauer, Defect groups in the theory of representations of finite groups, Illinois J. Math. 13 (1969), 53-73.

[4] R. Brauer, On 2-blocks with dihedral defect groups, in: Symposia Mathematica, Vol. XIII (Convegno di Gruppi e loro Rappresentazioni, INDAM, Rome, 1972), 367-393, Academic Press, London, 1974.

[5] M. Broué and J. B. Olsson, Subpair multiplicities in finite groups, J. Reine Angew. Math. 371 (1986), $125-143$.

[6] E. C. Dade, Blocks with cyclic defect groups, Ann. of Math. (2) 84 (1966), 20-48.

[7] J. Dietz, Stable splittings of classifying spaces of metacyclic p-groups, $p$ odd, J. Pure Appl. Algebra 90 (1993), 115-136.

[8] C. W. Eaton, Generalisations of conjectures of Brauer and Olsson, Arch. Math. (Basel) 81 (2003), 621-626.

[9] C. W. Eaton, R. Kessar, B. Külshammer and B. Sambale, 2-blocks with abelian defect groups, Adv. Math. 254 (2014), 706-735.

[10] C. W. Eaton and A. Moretó, Extending Brauer's Height Zero Conjecture to blocks with nonabelian defect groups, Int. Math. Res. Not. (to appear).

[11] K. Erdmann, Blocks of tame representation type and related algebras, Lecture Notes in Mathematics, Vol. 1428, Springer-Verlag, Berlin, 1990.

[12] W. Feit, The representation theory of finite groups, North-Holland Mathematical Library, Vol. 25, NorthHolland Publishing Co., Amsterdam, 1982.

[13] M. Fujii, On determinants of Cartan matrices of p-blocks, Proc. Japan Acad. Ser. A Math. Sci. 56 (1980), 401-403.

[14] S. Gao, On Brauer's k(B)-problem for blocks with metacyclic defect groups of odd order, Arch. Math. (Basel) 96 (2011), 507-512.

[15] S. Gao, Blocks of full defect with nonabelian metacyclic defect groups, Arch. Math. (Basel) 98 (2012), 1-12. 
[16] S. Gao and J. Zeng, On the number of ordinary irreducible characters in a p-block with a minimal nonabelian defect group, Comm. Algebra 39 (2011), 3278-3297.

[17] S. Hendren, Extra special defect groups of order $p^{3}$ and exponent $p^{2}$, J. Algebra 291 (2005), 457-491.

[18] L. Héthelyi, B. Külshammer and B. Sambale, A note on Olsson's Conjecture, J. Algebra 398 (2014), $364-385$.

[19] M. Holloway, S. Koshitani and N. Kunugi, Blocks with nonabelian defect groups which have cyclic subgroups of index p, Arch. Math. (Basel) 94 (2010), 101-116.

[20] H. Horimoto and A. Watanabe, On a perfect isometry between principal p-blocks of finite groups with cyclic p-hyperfocal subgroups, preprint.

[21] B. Huppert, Endliche Gruppen. I, Die Grundlehren der Mathematischen Wissenschaften, Band 134, Springer-Verlag, Berlin, 1967.

[22] I. M. Isaacs and G. Navarro, New refinements of the McKay conjecture for arbitrary finite groups, Ann. of Math. (2) 156 (2002), 333-344.

[23] R. Kessar, M. Linckelmann and G. Navarro, A characterisation of nilpotent blocks, arXiv:1402.5871v1.

[24] M. Kiyota, On 3-blocks with an elementary abelian defect group of order 9, J. Fac. Sci. Univ. Tokyo Sect. IA Math. 31 (1984), 33-58.

[25] S. Koshitani and N. Kunugi, Broué's conjecture holds for principal 3-blocks with elementary abelian defect group of order 9, J. Algebra 248 (2002), 575-604.

[26] S. Koshitani and H. Miyachi, Donovan conjecture and Loewy length for principal 3-blocks of finite groups with elementary abelian Sylow 3-subgroup of order 9, Comm. Algebra 29 (2001), 4509-4522.

[27] B. Külshammer, On p-blocks of p-solvable groups, Comm. Algebra 9 (1981), 1763-1785.

[28] K. Lux and H. Pahlings, Representations of groups, Cambridge Studies in Advanced Mathematics, Vol. 124, Cambridge University Press, Cambridge, 2010.

[29] G. Malle and G. Navarro, Inequalities for some blocks of finite groups, Arch. Math. (Basel) 87 (2006), 390-399.

[30] H. Nagao and Y. Tsushima, Representations of finite groups, Academic Press Inc., Boston, MA, 1989.

[31] J. B. Olsson, On 2-blocks with quaternion and quasidihedral defect groups, J. Algebra 36 (1975), 212-241.

[32] J. B. Olsson, Lower defect groups, Comm. Algebra 8 (1980), 261-288.

[33] L. Puig, Nilpotent blocks and their source algebras, Invent. Math. 93 (1988), 77-116.

[34] L. Puig and Y. Usami, Perfect isometries for blocks with abelian defect groups and Klein four inertial quotients, J. Algebra 160 (1993), 192-225.

[35] G. R. Robinson, Large character heights, Qd(p), and the ordinary weight conjecture, J. Algebra 319 (2008), 657-679.

[36] G. R. Robinson, On the focal defect group of a block, characters of height zero, and lower defect group multiplicities, J. Algebra 320 (2008), 2624-2628.

[37] B. Sambale, Fusion systems on metacyclic 2-groups, Osaka J. Math. 49 (2012), 325-329.

[38] B. Sambale, Brauer's Height Zero Conjecture for metacyclic defect groups, Pacific J. Math. 262 (2013), 481-507.

[39] B. Sambale, Further evidence for conjectures in block theory, Algebra Number Theory 7 (2013), 2241-2273.

[40] R. Stancu, Control of fusion in fusion systems, J. Algebra Appl. 5 (2006), 817-837.

[41] Y. Usami, On p-blocks with abelian defect groups and inertial index 2 or 3. I, J. Algebra 119 (1988), $123-146$. 
[42] A. Watanabe, Appendix on blocks with elementary abelian defect group of order 9, in: Representation Theory of Finite Groups and Algebras, and Related Topics (Kyoto, 2008), 9-17, Kyoto University Research Institute for Mathematical Sciences, Kyoto, 2010.

[43] S. Yang, On Olsson's conjecture for blocks with metacyclic defect groups of odd order, Arch. Math. (Basel) 96 (2011), 401-408.

Benjamin Sambale

Institut für Mathematik

Friedrich-Schiller-Universität

07743 Jena

Germany

benjamin.sambale@uni-jena.de 